\title{
Characterizing glitches and timing irregularities in pulsars and magnetars
}

\author{
Cristobal Espinoza ${ }^{1}$, Danai Antonopoulou ${ }^{2}$, Alessandro Patruno ${ }^{2}$, \\ Ben Stappers ${ }^{1}$ and Anna Watts ${ }^{2}$ \\ ${ }^{1}$ Jodrell Bank Centre for Astrophysics, University of Manchester, United Kingdom \\ email: cme@jb.man.ac.uk \\ ${ }^{2}$ Astronomical Institute Anton Pannekoek, University of Amsterdam, The Netherlands
}

\begin{abstract}
As the quantity and quality of timing data improves, we have reached the point at which the difference between timing noise and small glitches is unclear. As a consequence, the number of events reported as glitches which show unusual properties, quite different to those of giant glitches, has increased. For example, there is now a substantial population of glitches that apparently involve a decrease in spin-down rate rather than an increase. Motivated by the theoretical implications of such a result, we are conducting a detailed review of how glitches are detected and characterised. We have focused on three main questions: the observational biases affecting glitch detection; the methods used to characterise error bars on changes in spin and spin derivatives; and the physical mechanisms that could potentially explain the different populations of timing irregularities, in the light of improved characterisation. While glitches are thought to be a consequence of the internal dynamics of the star, magnetospheric processes may be responsible for other irregularities, as timing noise and peculiar glitch recoveries. We report the first results from this study, using a small sample of radio pulsars that exhibit a wide variety of glitching behaviour.
\end{abstract}

